\title{
One or Two Circular Shapes? A binary Detection for ECT Sensors
}

\author{
Yacine Oussar, Jérôme Lucas and Stéphane Holé \\ Laboratoire de Physique et d'Étude des Matériaux (LPEM) \\ PSL Research University, ESPCI Paris \\ Sorbonne Universités, UPMC Univ Paris 06 CNRS, UMR 8213 \\ 10 rue Vauquelin, 75231 Paris Cedex 05 - France
}

May 22, 2017

\begin{abstract}
Image reconstruction using ECT sensors rises several drawbacks. Recently, the authors proposed a novel methodology dedicated to a fast detection of the configuration: size and position, of a single circular form using basic algebraic operations. However, this technique is efficient when a single form is present in the sensor cross-section under interest. The present work proposes two different techniques that determine if the sensor cross-section contains one or two circular shapes. Both methods are applied to simulated measurements. They operate much differently and each comes with its own pros and cons. The first method considers that the sensor signal presents an axis of symmetry if a single circular object lies in the sensor. Otherwise, the symmetry is broken. It achieves a recognition rate of $73.9 \%$. In the second method, machine learning techniques are employed to perform a binary supervised classification. Promising rates of recognition over $99 \%$ are obtained. The present study also reveals that a 4-electrode sensor leads to the best recognition rates with both methods. This result was also established by the authors in a different framework when dealing with physical limitations on spatial resolution in ECT sensors.
\end{abstract}

\section{Introduction}

Electrical Capacitance Tomography (ECT) is a well known measurement technique. It is founded upon the principles of electrostatic $[6,7,8,9,10,11]$. Typically, an ECT sensor can yield information on the spatial configuration of a non-conducting material whose permittivity is different from that of the environment. Such situation is common when gas bubbles appear in a fluid. As a result, ECT is widely used for non destructive control of fluids conveyed in pipes. Generally, the shapes of typically non conducting bubbles present in a 
fluid are determined by solving an underdetermined set of linear equations using an iterative optimization [15]. This technique exhibits several drawbacks: the optimization is time consuming and the result may correspond to a numerical solution with no physical meaning.

Recently, the authors proposed a novel methodology dedicated to a fast detection of the configuration: size and position, of a single circular shape using basic algebraic operations [10, 11]. Instead of dealing with an image reconstruction founded on inverting an underdetermined set of equations by minimizing a cost function, the authors assume that a black-box inverse model that predicts the size and the position of single circular pattern exists and can be designed using data generated by numerical experiments. The black-box models are implemented using the LS-SVM technique [12]. Since the output of a LS-SVM model is computed using a set of basic arithmetic operations, this approach allows an almost instantaneous prediction of the pattern configuration which makes it highly attractive for a real-time implementation. However, this technique is efficient when a single shape is present in the sensor cross-section under interest. Its extension to the detection of several shapes is not straightforward. It is necessary to determine first the number of shapes present in the domain circumscribed by the sensor.

The present work proposes two different techniques that allow to determine whether the sensor cross-section contains one or two circular shapes. Both methods are applied to data obtained by numerical experiments. However, they operate much differently and each comes with its own pros and cons.

The first method is founded on the analysis of the circular symmetry of the sensor that is preserved if a single shape is present and often broken when a second shape with different size and position is also present in the sensor.

The second method proceeds by the implementation of a machine learning technique to separate the situations with one shape versus situations with two shapes. The detection of the number of shapes is treated as a classification problem.

The present paper begins by a recall of the capacitive sensing background and a presentation of sensors that use the electrical capacitive tomography. Thereafter, the problem statement is described. The two methods we propose are then described and the results obtained with both of them are given and discussed. The paper ends with a conclusion and a discussion on future work.

We are interested by ECT sensors formed by a set of electrodes arranged circularly. A typical ECT sensor formed by N electrodes is illustrated on Figure 1. The environment delimited by the sensor is characterized by a permittivity $\varepsilon_{1}$. A non conducting single circular shape with permittivity $\varepsilon_{2}$ lies in the closed region circumscribed by the sensor electrodes. This circular shape has unknown size and position respectively pointed by a radius $\mathrm{R}$ and two coordinates: $x$ and $y$. The measurement of the capacitances appearing between the sensor electrodes are performed by polarizing each electrode independently. When a voltage $V_{j}$ is applied to electrode $\mathrm{j}$, the measurement of the charge $Q_{i}$ that appears on electrode $i$, with a charge transfer circuit for instance [13], when all others are grounded, allows to determine the capacitive tensor $C$ according to 
the relation:

$$
\left[\begin{array}{c}
Q_{1} \\
Q_{2} \\
Q_{3} \\
\vdots \\
Q_{P}
\end{array}\right]=\left[\begin{array}{ccccc}
C_{11}=\sum_{j \neq 1} C_{1 j} & -C_{12} & -C_{13} & \cdots & -C_{1 P} \\
-C_{12} & C_{22}=\sum_{j \neq 2} C_{2 j} & -C_{23} & \cdots & -C_{2 P} \\
-C_{13} & -C_{23} & C_{33}=\sum_{j \neq 3} C_{3 j} & \cdots & -C_{3 P} \\
\vdots & \vdots & \vdots & \ddots & \vdots \\
-C_{1 P} & -C_{2 P} & -C_{3 P} & \cdots & C_{P P}=\sum_{j \neq P} C_{P j}
\end{array}\right]\left[\begin{array}{c}
V_{1} \\
V_{2} \\
V_{3} \\
\vdots \\
V_{P}
\end{array}\right]
$$

The capacitive sensor is symmetric. In addition, the diagonal components of the capacitive tensor are a linear combination of the other capacitances. As a result, there are at most $\frac{1}{2} P(P-1)$ independent capacitances. Usually, the image reconstruction consists in determining the permittivity distribution by solving an underdetermined set of linear equations obtained by a spatial disretization using the finite element method. Thus, an iterative algorithm $[15,5]$ is implemented to find a solution by minimizing a cost function.

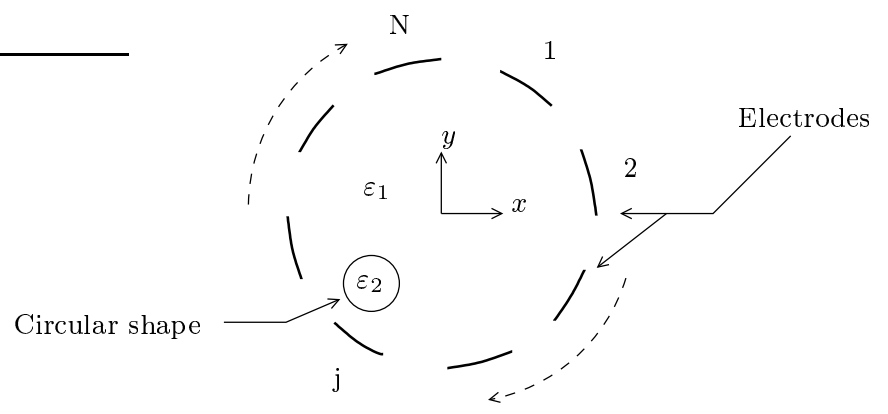

Figure 1: A N-electrode ECT sensor.

Recently, the authors proposed a novel approach to predict both size and position of a single circular shape lying in a ECT sensor using black-box inverse models and data generated by numerical experiments [11]. The black-box models are implemented using the LS-SVM technique. The output of a LS-SVM model is computed using a set of basic arithmetic operations. Thus, this method allows a fast prediction of the pattern configuration.

The training of the LS-SVM models was performed using data drawn from simulation. In fact, the simulation of electric fields is considered as very efficient. Thus, training sets were built using a knowledge-based model given by a set of differential equations. The spatial discretization of the sensor is implemented using the finite element modeling approach with the Gmsh software [3]. The differential equations were solved using GetDP software [2].

In addition to the fast prediction of the circular shape configuration, the proposed methodology allows to study the influence of the sensor architecture on the performances. In particular, the authors studied the impact of the number 
and the size of the electrodes. Figure 2 illustrates the prediction errors obtained with sensors formed by $2,3,4,5,6,8$ and 12 electrodes respectively. For all of them, the electrodes occupy $50 \%$ of the sensor perimeter as this covering ratio showed the best sensing performances. The results presented in this figure show that there is an optimal value for the number of electrodes. It is common for that kind of sensor [9]. Indeed the sensitivity of the sensor do not varies in the same way, inside the sensor, whether the object under detection is located close the the center or close to the electrodes.
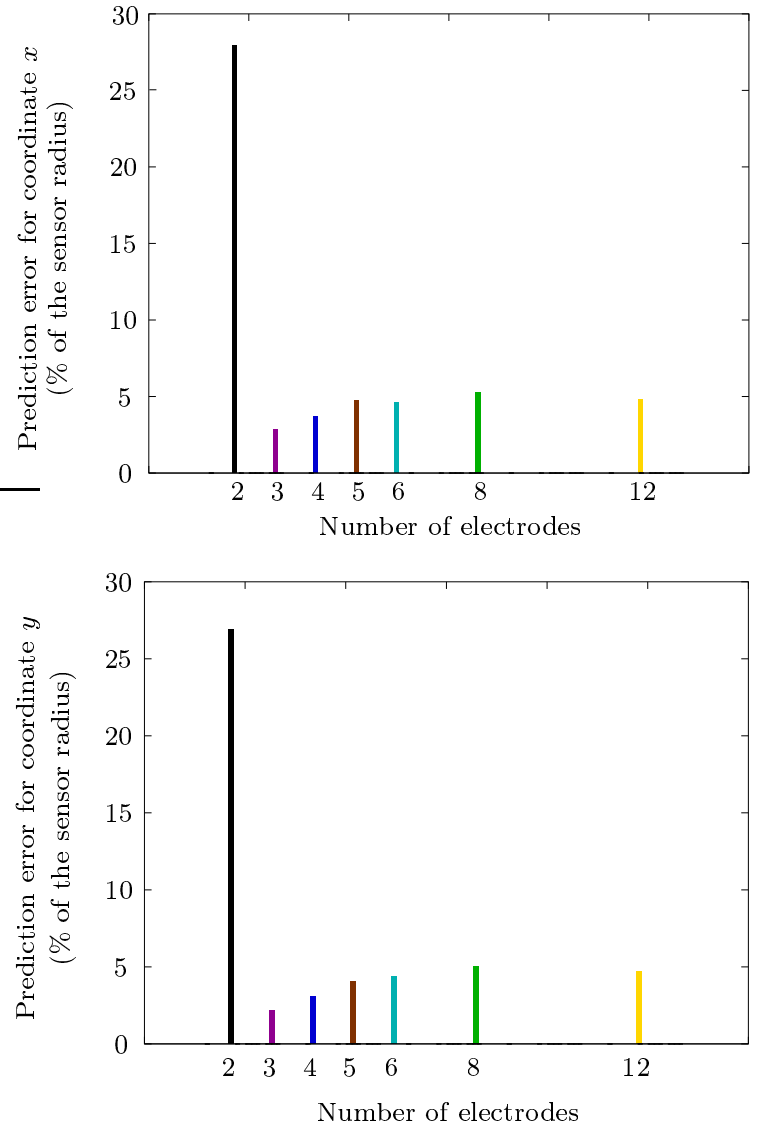

Figure 2: Prediction error for coordinates $x$ and $y$ in percentage of the sensor radius.

This approach offers satisfactory performances and is promising for real time applications. However, it also presents drawbacks: (i) it necessitates to form data sets for the design of nonlinear black box models, (ii) it is designed to detect a single shape. Thus, one can wonder what happens if more that one circular shape is present is the sensor domain. Numerical experiments showed that the method is unable to predict the size or the position neither of the first 


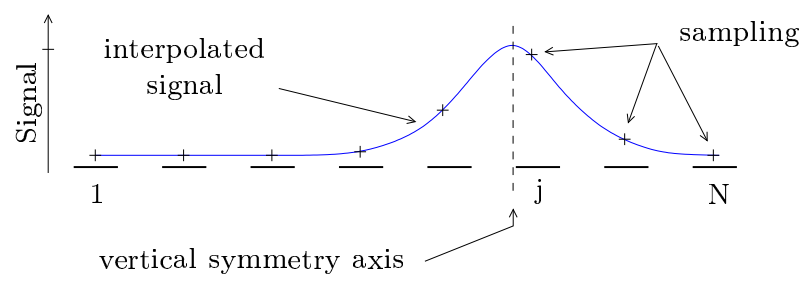

Figure 3: Aspect of the signal with one circular shape positioned as in figure 1.

shape nor of the second. In order to improve our method and to make it efficient to predict simultaneously the configuration of two circular shapes, we suggest to determine first if the closed region circumscribed by the sensor electrodes contains one or two circular shapes. In the following, we propose a study that presents two different techniques.

The first method operates by considering that the geometrical figure formed by the sensor including a single shape possesses an axis of symmetry. However, when a second circular shape with a different size and at a different position appears, the symmetry is most of the time broken. The first method aims to determine if an axis of symmetry exists in the signals provided by the capacitances of relation (1).

The second method, we propose, utilizes machine learning techniques to implement the detection of the number of shapes as a supervised classification problem. The capacitances measurements are considered as descriptors and used as inputs of support vector machines (SVM) classifiers whose binary outputs -1 and 1 point to one circular shape or two circular shapes respectively.

Both methods are not different by essence. They both use the same information from the capacitive tensor. The first one requires no learning phase and is simple. Machine learning techniques are more sophisticated and require a learning phase. They are known to be as efficient as possible for classification purposes.

\section{Direct symmetry based classification}

\section{$2.1 \quad$ Symmetry}

Considering figure 1, one can notice that because of the symmetry, and in absence of a circular shape, no electrode can be distinguished from the other. Consequently, without measurement noise, if one plots the $C_{i i}=\sum_{j \neq i}^{i \in\{1, . ., P\}} C_{i j}$ versus $i$, a perfectly flat curve is obtained. When there is a circular shape in the sensor, as soon as it is not perfectly centered, the precedent plot is no longer flat. The kind of plot that can be obtained is presented in figure 3

In this figure, the cross shaped markers are the measured values, that can be interpreted as a sampling of the blue plot in the same figure. The blue plot 
that we will refer as "unwound signal" in what follows, is the one that would be obtained for the considered circular shape by rotating the sensor around its center. This would be equivalent to shifting right of left the electrodes as in a logical register but in a continuous way and performing a measurement each time. The maximal value would be indeed be obtained in most cases depending of the relative circular shape radius and electrode sizes when there is an electrode in front of the circular shape[8]. In that case, the plot is no longer flat but presents a maximal value and the interpolated curve should be symmetrical considering both sides of its maximal value. The interpolated curve can be indeed be shifted indifferently right or left of any quantity without changing the symmetry because of the sensor circular geometry. To summarize, at this point, with one circular shape, the interpolated curve is whether symmetrical around its maximal value or a flat one.

In the case there are two circular shapes as in the situation depicted in figure 4 , the symmetry around the maximal value is broken. Note that it is broken even if the two circular shapes are the same and positioned in a symmetrical way with respect to the electrodes. In that case, there are two identical bumps in the unwound signal. There is indeed an axis of symmetry but it is not the axis that corresponds to one of maximal values

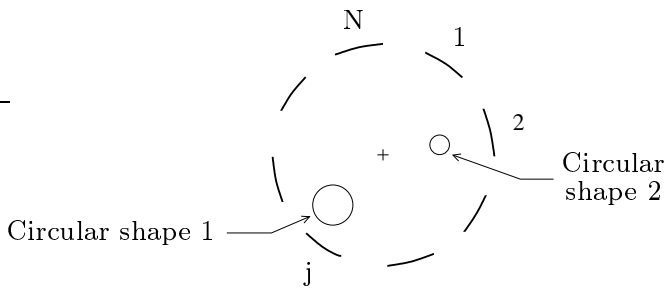

a)

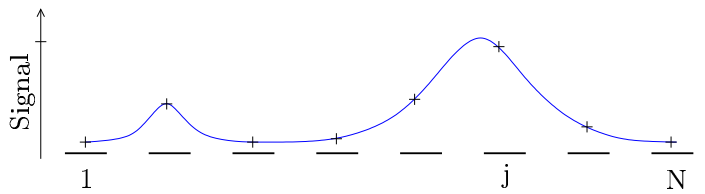

Figure 4: Aspect of the signal with two circular shapes.

\subsection{Criteria}

As presented in the precedent section, the symmetry around its maximal value of the sensor unwound signal yields information about the presence of a second circular shape in the sensor. There are two things to do at this point. Firstly obtain the unwound signal, secondly determine whether it is symmetrical around its maximal value.

The simplest way to estimate the unwound signal consists in the linear interpolation of the the measured signal. This method is simple enough but results 
in an unwound signal that will not be perfectly symmetrical even with one circular shape as in figure 3 . In this example the unwound signal maximal value will appear in front of the $\mathrm{j}^{\text {th }}$ electrode when it should be located a little bit on its left. Further more its derivative is not a continuous function which can be a drawback with some mathematical methods. To avoid this late impediment, it is also possible to use more sophisticated interpolation such as the so called cubic interpolation that respect the first order derivative continuity.

As a matter of fact, because of the circular geometry of the sensor, the unwound signal is periodical. Its period correspond to a full rotation of the sensor. Consequently, by duplicating the unwound signal, one obtains the periodic version of the unwound signal. Considering this property, using the FFT algorithm to interpolate the unwound signal seems a good candidate. This can be easily performed by padding zeros up to the required number of points in the FFT of the measured signal before going back to the unwound signal by calculating the inverse FFT.

In this work we have considered two criteria to estimate the symmetry of the unwound signal. The first one consists in using the FFT algorithm to estimate the symmetry of the unwound signal. Indeed considering the oddity property of the Fourier transform $\mathcal{F}$ :

$$
\mathcal{F}(\mathcal{F}(f(x)))=f(-x)
$$

one must have :

$$
\left\{x_{1}, \ldots, x_{N-1}\right\} \ominus F F T\left\{F F T\left\{x_{1}, \ldots, x_{N-1}\right\}\right\}=\{0, \ldots, 0\}
$$

In this equation the $\Theta$ sign refers to the element wise subtraction, and $\left\{x_{1}, \ldots, x_{N-1}\right\}$ is the sampled version of $f(x)$. The FFT acronym refers to an implementation of the Fast Fourier Transform algorithm where a $\frac{1}{\sqrt{N}}$ normalizing factor is included, thus avoiding additional normalization. The mean value of the left value in this equation defines $K_{1}$ the FFT symmetry criterion.

Finally $K_{1}$ the first criterion is calculated as:

$$
K_{1}=\left\langle\left\{C_{11}, \ldots, C_{P P}\right\} \ominus F F T\left(F F T\left\{C_{11}, \ldots, C_{P P}\right\}\right\}\right\rangle
$$

In this equation, the $\langle X\rangle$ denotes the average value of the components of vector $X$. Averaging renders the criterion somehow less dependent on the number of electrodes by allowing to yield comparable signal levels whatever this number. It is useful for instance when using a threshold with various number of electrodes.

In order to improve the performances of the detection of the two circular shapes situation, we used as an additional criterion which is the distance to the center of the "centroid" of the signal. The coordinates of the centroid $\left\{x_{c}, y_{c}\right\}$ can be calculated as: 


$$
\begin{aligned}
x_{c} & =\sum_{1}^{P} x_{i} C_{i i} / \sum_{1}^{P} x_{i} \\
y_{c} & =\sum_{1}^{P} y_{i} C_{i i} / \sum_{1}^{P} y_{i} \\
K_{2} & =\sqrt{x_{c}^{2}+y_{c}^{2}}
\end{aligned}
$$

Where $\left\{x_{i}, y_{i}\right\}$ are the coordinates of the center of electrode $i$. This criterion brings some additional information about the system. Indeed a symmetrical, with respect to the center of the sensor, distribution of circular shapes will yield a null $K_{2}$ criterion which is not the general case.

\subsection{Supervised classification}

Support Vector Machines (SVM) classification is a machine learning technique used to find an optimal separation between two classes: the maximum margin hyperplane [1]. The set of examples that are sufficient to determine the maximum margin hyperplane are called the support vectors. If the data are linearly separable, a linear SVM classifier is sufficient. Otherwise, if the data are not linearly separable, SVM classification proceeds by projecting the input vectors in a high dimensional space called the feature space then a linear separation is possible. In practice, this data conversion leads to the use of a kernel function. To be a SVM kernel, a function has to verify a set of conditions listed in [1]. An SVM discriminant function is given by:

$$
f(\mathbf{x})=\sum_{i=1}^{M} \alpha_{i} y_{i} k\left(\mathbf{x}, \mathbf{x}_{i}\right)+b,
$$

where: $k$ is the kernel function, $\mathbf{x}_{i}$ are the support vectors, $y_{i}$ are the corresponding class labels $( \pm 1)$ and $M$ is the number of support vectors. $\alpha_{i}$ and $b$ are the classifier parameters. They are adjusted during the training process.

As showed by relation 6, Support Vector Machines are linear-in-their-parameters models. This desirable property allow the training algorithm to converge to a unique solution. In addition, SVM come with a built-in regularization mechanism that confers to them the ability to design classifiers with good generalization capabilities. Thus, they consist of promising candidates to build efficient classifiers. In addition to SVM classifiers, two other data separation techniques were implemented in this study: the traditional Least Squares (LS) method, and the K-Nearest Neighbor algorithm (K-NN) [4].

\section{How data was generated}

The axis of symmetry method as well as the design of classifiers work on data. The data can be generated using either a real experimental setup or numerical 
experiments. Since a large number of real experiments is complicated to carry out, we preferred the numerical alternative. Indeed, the simulation of electric fields is considered as very efficient. As mentioned before, both the number and the size of electrodes are considered as relevant parameters of the the sensor architecture. In $[9,11]$ it was proved that ECT sensors with electrodes covering $50 \%$ of their perimeter show better spatial resolution. Thus, in our simulations, data was generated using ECT sensors formed by 2 to 6 electrodes covering $50 \%$ of the sensor perimeter. For every sensor architecture, 1000 different situations were randomly drawn. In each situation, 2 circular shapes lie inside the sensor. The shapes radii are pulled in the range $[0.1 ; 0.45]$. The coordinates $x$ and $y$ of the shapes centers are pulled in the range $[-0.8 ; 0.8]$. When a situation is generated, it is first verified if it corresponds to a realistic configuration. In particular, the circular shapes must be totally inside the sensor and should not be contiguous. If a situation is not compatible with theses conditions, it is rejected and another is drawn. For all the satisfactory situations, the capacitances are calculated by polarizing each electrode to $1 \mathrm{~V}$ when the others are grounded. Note that each situation corresponds to a different mesh from the FEM point of view. We considered circular forms of air with relative permittivity $\varepsilon_{2}=1$ in an oil flow having a relative permittivity $\varepsilon_{1}=3$.

\section{Results}

\subsection{Results with the axis of symmetry method}

Figure 5 presents the distribution of the one and two bubbles situations in the $K_{1} K_{2}$ data space. The results presented where obtained with a simple linear interpolation to obtain the unwound signal. One can see that the one circular shapes situation leads to $K_{1}$ criterion most of the time below 0.1 . unfortunately, the two circular shapes do not lead systematically lead to greater values of this criterion which does not allow to completely distinguish the two situations. The efficiency of the center of mass criterion is not better. We do not pretend at this point that the criterion proposed is the best that can be obtained, but it well illustrates the kind of criterion that can be thought of when using physical considerations. The overall classification efficiency of this method is about $80 \%$.

At this point, one may think of refining the criterion to obtain better result. Indeed the known sensitivity map of the kind of sensors [8] along with this analysis of the "fail" cases yields new hints and ideas. By doing so, better results can indeed be obtained but it consist in blind try and test work. In this work we present instead, as a comparison, a more systematic method based on supervised classification.

\subsection{Results with the supervised classification}

As mentioned above, 1000 different situations were simulated. The whole available data was divided into two sets: a training set formed by 700 examples and 


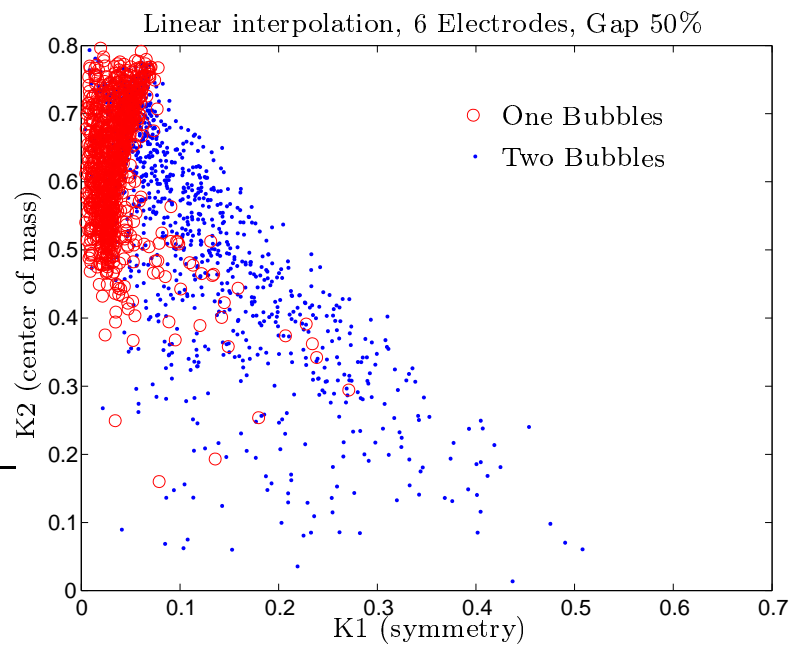

Figure 5: Distribution of the 1 or two circular shapes situation using the criterions

a validation set formed by 300 examples. Thus, for all the classification methods we implemented, the classifiers were selected using a validation procedure that consisted in using a single validation set. Table 1 illustrates the recognition rates in percentage obtained on the validation set for the five different sensor architecture we consider. Sensors formed by 2 or 3 electrodes do not provide sufficient information to recognize if one or two circular shapes are present in the sensor environment. A comparison of the results obtained with both Least Squares and Linear SVM methods suggest that data is no linearly separable. Indeed, the performances do not improve whatever the sensor architecture. Sensors formed by 4 electrodes or more allow the design of efficient classifiers with other techniques. In particular, the 1-NN method permits to obtain a recognition rate of $86.2 \%$ on the validation set using a 5 -electrode sensor and the Nonlinear SVM method allows a performance of $99.5 \%$.

\begin{tabular}{|c|c|c|c|c|c|}
\hline Technical classification & \multicolumn{7}{|l|}{ Number of electrodes } \\
\hline & 2 & 3 & 4 & 5 & 6 \\
\hline 1-NN & 57.3 & 62.3 & 82.3 & 86.2 & 85.3 \\
\hline K-NN $(K)$ & $64(25)$ & $69.8(15)$ & $82.5(3)$ & $86.2(1)$ & $85.3(1)$ \\
\hline Least Squares & 52.7 & 49.2 & 66.8 & 63.3 & 66.7 \\
\hline Linear SVM & 55.5 & 52.2 & 69 & 67.5 & 66.5 \\
\hline Nonlinear SVM & 65.5 & 71.8 & 98.8 & 98.5 & 99.5 \\
\hline
\end{tabular}

Table 1: Recognition rates in percentage on the validation set.

Overall, classifiers designed with nonlinear support vector machines show promising performances. Whereas the recognition rate is close to $100 \%$, this 


\begin{tabular}{|c|c|c|c|c|c|}
\hline Noise level & $0 \%$ & $5 \%$ & $10 \%$ & $15 \%$ & $20 \%$ \\
\hline \hline Recognition rate & 99.5 & 98.8 & 97.7 & 96 & 93.9 \\
\hline
\end{tabular}

Table 2: Recognition rates in percentage of the validation set in presence of noise.

goal can not be considered as realistic. Indeed, in a real world implementation, a noise may act on the measurements of the capacitances. Consequently, the performance may degrade slightly. Figure 6 illustrates graphically the recognition rates detailed on Table 1 .

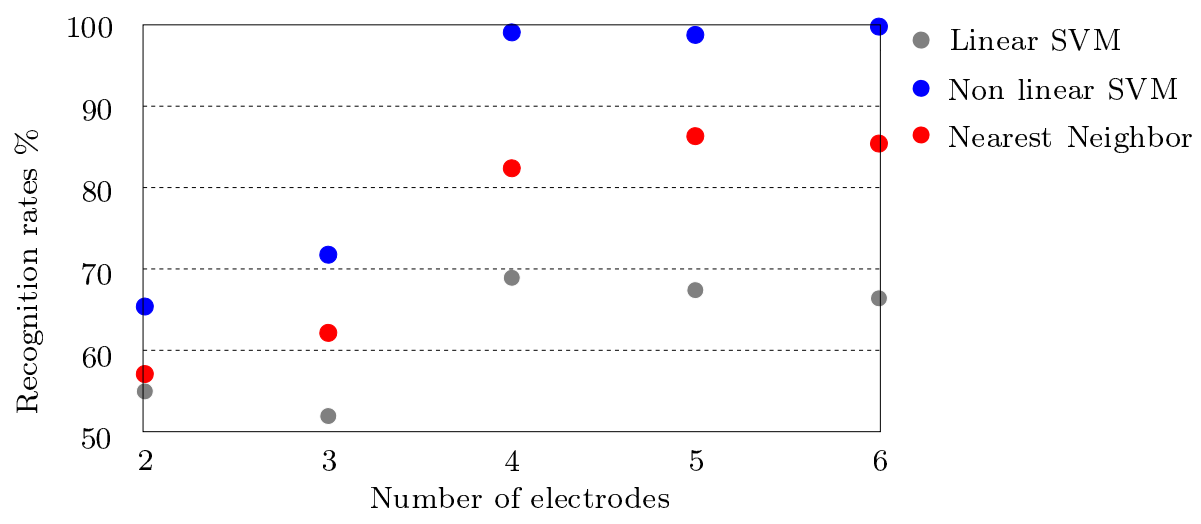

Figure 6: Recognition rates achieved by the 3 methods: Linear SVM, Nonlinear SVM and K-Nearest Neighbor.

\section{Noise robustness}

The results presented above were obtained using noise free data. If data was drawn from real measurements, it would be sullied by noise. This may lead to a decrease in the recognition rates. One can wonder how does the presence of noise in the data affect the performance of the supervised classification techniques implemented in this study. Several numerical experiments were conducted. A time and space varying noise was introduced in the validation set as realizations of a uniformly distributed random variable in the interval $[-a, a]$. Four different noise levels were considered corresponding to four different values of $a$ : $5 \%$, $10 \%, 15 \%$ and $20 \%$ of the average signal amplitude. These noise levels are those encountered at the laboratory on equivalent ECT systems [14].

Table 2 illustrates the recognition rates obtained on the same validation set of Table 1 with classifiers implemented using Nonlinear SVM. This technique was selected since it leads to the best performance. Since the noise is a realization of a uniformly distributed random variable, its effect varies from a realization to another. Thus, for every noise level, one hundred different realizations were 


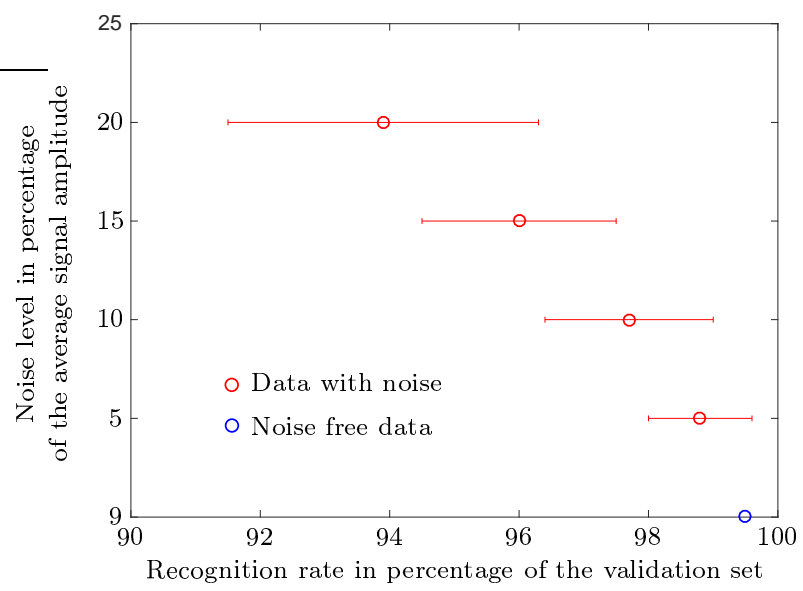

Figure 7: Recognition rates and their domains of variation for 100 different realizations of noise.

performed. The circles on Figure 7 correspond to the rate means over the 100 experiments also given on Table 2. The intervals give the domain covered by the obtained 100 rates.

As expected, the recognition rate goes down when a noise acts on data. For a noise level corresponding to of $10 \%$ the average signal or less, the decrease in the classifier performance remains moderate. Such a noise level can be considered as realistic. For noise levels beyond $10 \%$, the loss in the performance becomes significant. However, one can consider those levels as quite exceptionally. Note that the worst recognition rate obtained with a noise level of $20 \%$ is $91.3 \%$. It remains much better than all those obtained with the other classification techniques even with noise free data.

\section{Conclusion}

Two different approaches to determine whether one or two circular shapes are lying in an ECT sensor were implemented. Both approaches use capacitance measurements drawn from numerical experiments.

The first approach is founded on the search of an axis of symmetry. It is a direct method in the sense that it does not require the design of a filter. It possesses the advantage of flexibility and achieves a recognition rate of about $80 \%$.

The second approach consists in designing supervised classifiers using machine learning techniques. It is an indirect approach in the sense that it necessitates the availability of data and the design of classifiers. Several classification methods were implemented. Results show that data is not linearly separable and nonlinear Support Vector Machines achieved promising recognition rates 
up to $99 \%$.

This work illustrates, on an example, the efficiency of machine learning techniques with capacitive sensors to take advantages of data as soon as more "physically based" techniques do not yield straightforwardly the expected results.

\section{References}

[1] Cristianini N., Shawe-Taylor J. Support Vector Machines and other Kernelbased Learning Methods, Cambridge University Press, 2000.

[2] GetDP Software. Available online. http://getdp.info/

[3] Gmsh Software. Available online. http://gmsh.info/

[4] Hastie T., Tibshirani R., Friedman J. The Elements of Statistical Learning, Springer, New-York, 2009.

[5] J. Jang and S. Lee and K. Kim and B. Choi, Modified Iterative Landweber Method in Electrical Capacitance Tomography, Measurement Science and Technology, Vol. 17, 2006.

[6] L. K. Baxter, Capacitive Sensors Designs and Applications, Wiley-IEEE Press, 1996.

[7] R. Puers, Capacitive sensors: when and how to use them, Sensors Actuators A, Vol. 37-38, pp. 93-105, 1993.

[8] J. Lucas, S. Hole, and C.Batis, Analytical capacitive sensor sensitivity distribution and applications, Measurement Science and Technology, Vol. 17, pp. $2767-2478,2006$.

[9] J. Lucas, C. Margo, Y. Oussar, S. Holé, Physical Limitations on Spatial Resolution in Electrical Capacitance Tomography, Measurement Science and Technology, Vol. 26, doi:10.1088/0957- 0233/26/12/125105, 2015.

[10] Y. Oussar, C. Margo, J. Lucas, S. Holé, ECT Sensor Design using Machine Learning Techniques, In Proceedings of the 2012 Electrostatics Joint Conference, Cambridge (Canada), June 2012.

[11] Y. Oussar, C. Margo, J. Lucas, S. Holé, Fast Circular Shapes Detection with Optimal Design of ECT Sensors, COMPEL - The International Journal for Computation and Mathematics in Electrical and Electronic Engineering, in press.

[12] V. Vapnik, The Nature of Statistical Learning Theory, Springer, New York, 1995.

[13] S Buchman., J Mester., T J Summer, Electrical measurement, signal processing, and displays, Chapter 8, CRC Press LLC, ISBN 0-8493-1733-9. 
[14] C. Margo, J. Lucas, T Ditchi, E. Géron, S.Holé, and J. Lewiner, Wood-chip Water Content Sensor with Capacitive Tomography, The 10th International Symposium Of Measurement Technology And Intelligent instruments, July 2011.

[15] W. Q. Yang and L. Peng, Image Reconstruction Algorithm for Electrical Capacitance Tomography, Measurement Science and Technology, Vol. 14, pp.R1-R13, 2003. 